has enabled the required FMT infrastructure to be developed to support prospective FMT research in the UK.

\section{OTH-05 A REVIEW FOLLOWING A DR FOSTER'S REPORT OF APPARENTLY HIGH GASTROINTESTINAL HAEMORRHAGE MORTALITY}

'Duncan Napier*, 'Kate Hale, ${ }^{2}$ Hazel Woodland, ${ }^{1}$ Richard Makins. ${ }^{1}$ Cheltenham General Hospital, Cheltenham, UK; ${ }^{2}$ Bristol Royal Infirmary, Bristol, UK

\subsection{6/gutjnl-2019-BSGAbstracts.424}

Introduction Dr Foster publishes annual mortality rates for every hospital trust and compares these across the NHS. Mortality rate indicators are mechanisms by which the NHS and hospital trusts can identify potentially poorly performing services to facilitate investigation and change. Data is collected from Hospital Episode Statistics (HES) which relies on accurate coding from the first two episodes of care within an hospital and linked to Office for National Statistics data. Dr Foster produced a report looking at mortality at Gloucestershire Hospitals NHS Foundation Trust between October 2015 and September 2016, whereby mortality from gastrointestinal haemorrhage $(\mathrm{GH})$ was statistically higher than expected. This review sought to evaluate the accuracy of the Dr Foster report.

Methods Data submitted to HES via Secondary Uses Services, used in the creation of the Dr Foster report was analysed. Hospital records were reviewed for each patient for the period leading up to their death. Admission diagnosis was recorded as determined by clinical documentation from the first two or three episodes of care. Cause of death was determined by review of death certificates or contact with the regional Coroner's office. Subsequently, all patients with a coding diagnosis relevant to $\mathrm{GH}$ during the study period were listed and notes were reviewed of those who died within or up to 30 days after the study period. The confirmed diagnosis and cause of death were compared with the coding diagnosis from admission documentation.

Results 49 patients were reported to have died from GH compared to an expected number of 35.5 according to the $\mathrm{Dr}$ Foster report, 1 of which was a duplicate. 43 notes were reviewed (5 incomplete or unavailable) and only 32 of these had a differential diagnosis of $\mathrm{GH}$ within the first 3 clinical episodes. However, only 12 of these ultimately had GH listed as a cause of death on the patient's death certificate. On subsequent analysis of all 61 patients who died with a code suggestive of $\mathrm{GH}$ during their admission, 39 had a differential diagnosis of $\mathrm{GH}$ within the first three episodes.

Conclusions Dr Foster relies on accurate coding and a correct initial diagnosis to provide robust data on hospital mortality. Our study shows that coding within the study was often inaccurate but more importantly that the initial diagnosis (within the first few clinical episodes) often did not reflect the confirmed diagnosis after specialist review and investigations were completed. In addition, patients' death certificates recorded a cause of death unrelated to $\mathrm{GH}$ in many cases, the commonest being bronchopneumonia. Admission diagnosis often fails to reflect the actual admission diagnosis or cause of death and can result in inaccurate mortality statistics.

\section{OTH-06 OUTCOMES FROM THE FIRST FORMALISED NATIONAL ENDOSCOPY SERVICE IN SIERRA LEONE}

${ }^{1}$ DE Thomas-Macauley ${ }^{*},{ }^{1} S$ Conteh, ${ }^{1} \mathrm{~F}$ Ngongo, ${ }^{2} \mathrm{~J}$ Hancock, ${ }^{3} \mathrm{C}$ Mountford, ${ }^{3} \mathrm{DL}$ Nylander, ${ }^{2} \mathrm{CW}$ Wells, ${ }^{2} \mathrm{R}$ Bevan . ${ }^{1}$ Choithram Memorial Hospital, Freetown, Sierra Leone; ${ }^{2}$ North Tees and Hartlepool NHS Foundation Trust, UK; ${ }^{3}$ Newcastle Upon Tyne Hospitals NHS Foundation Trust, UK

\subsection{6/gutjnl-2019-BSGAbstracts.425}

Introduction Sierra Leone is a developing country in Africa, with a population of 7 million. Prior to 2016 there was no recognised endoscopy service in the country, although one hospital in the capital Freetown (pop. 1 million) owned an endoscopy stack. Medical services are rudimentary, but with a small group of motivated doctors and a grant from the BSG, a training programme was developed to support the provision of endoscopy.

Here we describe the outcomes from the service since it's inception in December 2016.

Methods 3 endoscopists have been trained in OGD, and are supported remotely by 5 clinicians in the UK via Whatsapp. An annual training visit has been undertaken to consolidate skills. Weekly endoscopy lists are delivered in Choithram Hospital, with reports generated on ADAM endoscopy reporting software. An Excel spreadsheet database of all procedures is maintained by the endoscopists.

The database was interrogated for procedures since the start of the service up to the end of January 2019. Data were collected on gender, age, intubation of the second part of the duodenum, length of procedure, CLO test results and endoscopic findings.

Results 289 procedures have been performed. 56\% were male, median age 43 years (range 1-8). The number of procedures increased significantly in the second year of the service. The most frequent referral reason is dyspepsia (62\%)

Overall results are shown in table 1.

Pathology detection rates have been similar in each year of the programme. 18 cancers were identified, 25 cases with ulcers, and 21 cases with evidence of portal hypertensive changes (oesophageal/gastric varices, or portal gastropathy).

\begin{tabular}{|c|c|c|c|}
\hline & $\begin{array}{l}\text { December } 2016 \text { - end } \\
\text { December } 2017\end{array}$ & $\begin{array}{l}\text { January } 2018 \text { - end } \\
\text { January } 2019\end{array}$ & Total \\
\hline Procedures & 74 & 215 & 289 \\
\hline CLO test performed ${ }^{*}$ & 3 & 163 & 166 \\
\hline +ve result (\%) & $1(33)$ & $112(69)$ & $113(68)$ \\
\hline D2 reached $(\%)^{*}$ & $64 / 73(88)$ & 192/202 (95) & $\begin{array}{l}256 / 275 \\
(93)\end{array}$ \\
\hline $\begin{array}{l}\text { Mean procedure length, } \\
\text { mins }(S D)^{*}\end{array}$ & $31(16.4)$ & $19(8.5)$ & $\begin{array}{l}22 \\
(12.0)\end{array}$ \\
\hline Pathology detected $(\%)^{*}$ & $55 / 72(76)$ & $155 / 203(75)$ & $\begin{array}{l}210 / 275 \\
(76)\end{array}$ \\
\hline
\end{tabular}

*complete data not available for all procedures - denominator given in each case

Discussion Sierra Leone now has a functioning upper GI endoscopy service. This service is providing high quality procedures, with overall D2 intubation rate meeting the JAG requirement of 95\%. Pathology detection rates are high, possibly due to current practice of only those with the most severe symptoms being referred. Clinically important lesions (ulcers, cancers and 
complications of cirrhosis) are being detected, allowing patient care to be adjusted in light of these findings. As the service has developed, referral numbers have increased due to increased awareness and positive reputation developed by the service. The procedures have improved in quality, with a rising D2 intubation rate and a falling duration of procedure. These data demonstrate that an endoscopy service can be successful delivered, using innovative teaching methods and ongoing support delivered by a UK team. The endoscopists are now delivering training to new trainees, with a view to self sufficiency in training and expansion to other areas in Sierra Leone.

\section{OTH-07 IDENTIFICATION OF IBD COHORTS FROM LINKED ENDOSCOPY AND HISTOLOGY REPORTS USING NATURAL LANGUAGE PROCESSING}

1Jonathan Brown*, '2Sebastian Zeki. 'Gloucestershire Hospitals Nhs Trust, Gloucester, UK; ${ }^{2}$ Gastroenterology Data Sciences Institute, St Thomas' Hospital, London SE1, UK

\subsection{6/gutjnl-2019-BSGAbstracts.426}

Introduction Patients with inflammatory bowel disease (IBD) are likely to undergo multiple lifetime endoscopic procedures which generate histopathological reports. Managing these patients requires clinicians to derive a phenotypic overview from numerous episodes and diverse sources which can be time consuming, incomplete and subjective. We set out to evaluate the potential for a computer to extract phenotypic parameters from a series of linked histopathology and endoscopy reports to characterise an IBD cohort.

Methods 118,108 lower GI endoscopic procedure reports (200-017) and 62,051 lower GI histology reports (200-017) from GRH were imported into an SQL database. Unique patient identification numbers from the merged dataset were replaced with 128 bit hexadecimal GUIDs and all patient identifiable information subsequently stripped from the data tables (Service Evaluation Project 8622).

Text processing was undertaken in Python pandas dataframes:

1) Import both datasets and separate all words by single space, convert to lower case, remove apostrophes

2) Correct spelling of key words using Levenshtein distance

3) Find regular expressions that match disease phenotypes

4) Exclude non-IBD colitis diagnoses

5) Exclude negated IBD diagnoses

6) Export tagged machine interpreted reports back to SQL database

7) Select 100 random reports for each IBD confirmed or negated diagnosis to validate against original text

8) Return to steps - to modify regular expression reference lists to improve sensitivity and specificity.

Results The following results were obtained after multiple validation cycles initially based on an empiric regular expression dataset.

\begin{tabular}{lll}
\hline & Sensitivity & Specificity \\
\hline Procedure indication: Previously known IBD colitis & $100 \%$ & $100 \%$ \\
Procedure indication: Previously known Crohn's & $100 \%$ & $100 \%$ \\
disease & & \\
Histopathological interpretation: IBD colitis & $100 \%$ & $98 \%$ \\
Histopathological interpretation: IBD Crohn's & $100 \%$ & $98 \%$ \\
\hline
\end{tabular}

Some caution is required in interpretation of the specificity of the Crohn's and ulcerative colitis histopathology reports. Many samples are described as showing features of both diseases and the final conclusion is given as a likelihood or unclassified. The specificities reported here are for all IBD and do not reflect a capacity to distinguish between the different types.

Conclusions The evolution of the disease characteristic regular expressions through repeated validation cycles has provided a powerful tool for the automated generation of IBD databases from text in semi-structured endoscopy and histology reports. The potential for the scheduling of surveillance and linkage to other systems, such as primary care prescribing, are obvious. Further development will include a more detailed phenotypic interpretation and computation of the histopathological certainty in distinguishing the types of IBD.

\section{OTH-11 INTELLIGENT LIVER FUNCTION TESTING (ILFT) IN ACTION}

${ }^{1}$ Emma Robinson*, ${ }^{2}$ Jennifer Nobes, ${ }^{1}$ Paul Brennan, ${ }^{2}$ Ellie Dow, ${ }^{1}$ John Dillon. ${ }^{1}$ University of Dundee, Dundee, UK; ${ }^{2}$ NHS Tayside, Dundee, UK

\subsection{6/gutjinl-2019-BSGAbstracts.427}

Background and aims Liver Function Tests (LFTs) are commonly requested and are abnormal in $20 \%$ of cases. Intelligent liver function testing (iLFT) aims to improve diagnostic proficiency and quality of investigation thereby reducing overall costs to practitioners and patients and subsequent secondary care referrals. Following a pilot trial, iLFT was rolled out across general practices in NHS Tayside from August 2018.

Method The automated iLFT algorithm uses the combination of clinical features (alcohol consumption, BMI and metabolic syndrome), diagnostic criteria for liver disease, an investigation ordering and reporting system, and the tracked blood sciences system to generate a diagnosis or descriptor of the abnormality with fibrosis staging.

Management plans are disseminated back to the GP, along with a recommendation for one of three outcomes; a) secondary care follow up for advanced liver disease or complex treatment, b) primary care follow up of early or simple liver disease, c) where a clear diagnosis is unclear; the GP receives staging and prognostic information including referral criteria. Results of iLFT in action, over 6 months were analysed.

Results 777 iLFT requests over 6 months to 31/01/19. 568/ 777 requests had at least 1 abnormal LFT triggering the iLFT cascade. $169(29.8 \%)$ were referred to secondary care, 399 (70.2\%) patients were investigated further or managed in primary care. The most common finding was isolated ALT elevation without fibrosis (24.8\%), followed by alcohol related liver disease without fibrosis(16.4\%) then non-alcoholic fatty liver disease with fibrosis (8.1\%) and elevated ALT and GGT without fibrosis (8.1\%). There were $2 \mathrm{HBV}$ infections, 8 HCV infections, 3 haemochromatosis diagnoses, $1 \mathrm{PBC}$ and 1 A1AT PiS variant (risk of lung/liver disease) and 17 carriers of A1AT S or Z variant.

Conclusion Using iLFT; serious liver disease has been detected and referred to secondary care. High numbers of LFTs have been investigated readily and management plans generated. Of all the patients with abnormal LFT results only a third have 